\title{
Improved Mutual MRAS Speed Identification Based on Back-EMF
}

\author{
Hong Zheng ${ }^{\dagger}$, Jiancheng Zhao* and Liangzhong Liu*
}

\begin{abstract}
In the design of sensorless control system for induction motor, high-precision speed estimation is one of the most difficult problems. To solve this problem, the common method is model reference adaptive method (MRAS). MRAS requires accurate motor parameters to estimate rotor speed precisely. However, when motor is running, the variety of temperature and magnetic saturation will lead to the change of motor parameters such as stator resistance and rotor resistance, which will lower the accuracy of the speed estimation. To improve the accuracy and rapidity of speed estimation, this paper analyses the mutual MRAS speed identification based on rotor flux linkage, and proposes an improved mutual MRAS speed identification based on back-EMF. The improved method is verified by Simulink simulation and motor experimental platform based on DSP2812. The results of simulation and experiment indicate that the method proposed by this paper can significantly improve the accuracy of speed identification, and speed up the response of identification.
\end{abstract}

Keywords: Sensorless control, MRAS, Mutual identification, Speed estimation, Motor experimental platform

\section{Introduction}

In order to improve the static and dynamic performance, field oriented vector control or direct torque control is commonly used to motor drives system. Its speed feedback is usually obtained from speed sensor. In the traditional way of control by vector control system, the use of speed sensors would increase the cost of system, decrease system reliability and narrow the application range of the system. However, sensorless vector control system can reduce the amount of system devices, low cost and improve reliability of the system [1]. Therefore, sensorless control algorithm has been widely studied by scholars and researchers in recent decades.

At present, MRAS-based speed identification is the most mature sensorless speed estimation algorithm. Which requires motor parameters directly or indirectly to calculate the speed feedback. However, motor parameters will change with temperature and flux saturation, such as the resistance of stator and rotor. The change of these two parameters will lead to reduce the accuracy of rotor speed estimation. So when estimating the speed, it is necessary to identify the stator and rotor resistance online at the same time.

Because the mutual speed identification based on rotor flux linkage MRAS (RF-MRAS) cannot eliminate the influence of the integral element [2], this paper proposes an improved mutual speed estimation method based on backEMF MRAS (BEMF-MRAS). Meanwhile, the feasibility

$\dagger$ Corresponding Author: Dept. of Automation, University of Electronic Sci\&Tech of China, China. (macrozheng@uestc.edu.cn)

* Dept. of Automation, University of Electronic Sci\&Tech of China, China. (\{zjclyc, uestc liu\}@qq.com)

Received: July 26, 2014; Accepted: December 26, 2015 and practicality of this algorithm is verified by the results of Simulink simulation and motor experimental platform.

\section{Mutual Speed Identification Based on MRAS}

The basic principle of MRAS: systems can be described as mathematical model but not all the parameters or variables can be fully measured, the output error between reference model and adjustable model can be used to design an adaptive mechanism. Meanwhile, one or more parameters (or unmeasured variables) of the system can be regulated to make the output error between reference model and adjustable model be zero.

The theory of mutual MRAS can be summed as follows [3]: Define two different function sets A and B, which have the same function range. If sets $\mathrm{B}$ contains $m$ parameters but sets A does not, sets A can be used as the reference model while sets $\mathrm{B}$ as the adjustable model. The $m$ parameters can be identified through adaptive law. However, if sets A contains n parameters but sets B does not, sets B can be used as the reference model while sets $\mathrm{A}$ as the adjustable model. Meanwhile, the $n$ parameters also can be identified through adaptive law. If all the $m+n$ parameters are the values to be estimated, we can identify these parameters at the same time through mutual identification.

\subsection{Stator resistance and speed identification based on BEMF-MRAS}

Induction motor voltage /current model based on backEMF respectively: 


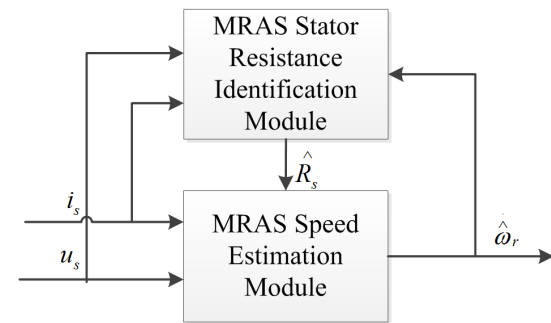

Fig. 1. Block diagram of stator resistance and speed estimation

$$
\begin{gathered}
{\left[\begin{array}{c}
e_{r \alpha} \\
e_{r \beta}
\end{array}\right]=\frac{L_{r}}{L_{m}}\left[\begin{array}{c}
u_{s \alpha} \\
u_{s \beta}
\end{array}\right]-\frac{L_{r}}{L_{m}}\left[\begin{array}{cc}
R_{s}+\sigma L_{s s} p & 0 \\
0 & R_{s}+\sigma L_{s} p
\end{array}\right]\left[\begin{array}{c}
i_{s \alpha} \\
i_{s \beta}
\end{array}\right]} \\
{\left[\begin{array}{c}
\dot{e}_{r \alpha} \\
\dot{e}_{r \beta}
\end{array}\right]=\left[\begin{array}{cc}
-\frac{1}{T_{r}} & -\omega_{r} \\
\omega_{r} & -\frac{1}{T_{r}}
\end{array}\right]\left[\begin{array}{c}
e_{r \alpha} \\
e_{r \beta}
\end{array}\right]+\frac{L_{m} p}{T_{r}}\left[\begin{array}{c}
i_{s \alpha} \\
i_{s \beta}
\end{array}\right]}
\end{gathered}
$$

In Eq. (1) and (2), $e_{r \alpha}=\frac{d \psi_{r \alpha}}{d t}, e_{r \beta}=\frac{d \psi_{r \beta}}{d t}$, which is the back-EMF on static coordinate system. Eq. (1) is called voltage equation and Eq. (2) is called current equation in the paper. As shown, voltage equation based on backEMF contains stator resistance $\mathrm{R}_{\mathrm{s}}$, but does not contain rotor speed $\omega_{r}$, while current equation based on back-EMF contains rotor speed $\omega_{r}$, but does not contain stator resistance $R_{s}$. Based on the theory of mutual MRAS, it takes current equation as a reference model and selects voltage equation as an adjustable model to identify the stator resistance. Similarly, it takes voltage equation as a reference model and chooses current equation as an adjustable model to estimate rotor speed, as shown in Fig. 1.

In Fig. 1., the self-adaptive laws of rotor speed is:

$$
\hat{\omega}_{r}=\left(K_{p}+\frac{K_{i}}{s}\right)\left(e_{r \beta} \hat{e}_{r \alpha}-e_{r \alpha} \hat{e}_{r \beta}\right)
$$

In Eq. (3), $\hat{\mathrm{e}}_{r \alpha}$ and $\hat{\mathrm{e}}_{r \beta}$ is the rotor back-BEF of the adjustable model using current equation, $\mathrm{e}_{r \alpha}$ and $\mathrm{e}_{r \beta}$ is the rotor back-BEF of the reference model using voltage equation.

The self-adaptive law of stator resistance is:

$$
\hat{R}_{s}=\left(K_{p}+\frac{K_{i}}{s}\right)\left(e_{r \alpha} \hat{e}_{r \beta}-e_{r \beta} \hat{e}_{r \alpha}\right)
$$

In Eq. (4), $\hat{\mathrm{e}}_{r \alpha}$ and $\hat{\mathrm{e}}_{r \beta}$ is the rotor back-BEF of the adjustable model using voltage equation, $\mathrm{e}_{r \alpha}$ and $\mathrm{e}_{r \beta}$ is the rotor back-BEF of the reference model using current equation.

By choosing different reference model, mutual MRAS identifies rotor speed and stator resistance respectively through different loops, so it can independently identify rotor speed and stator resistance online. Meanwhile, as the Eq. (3), (4) shown, the influence of the integral element caused by RF-MRAS is eliminated by using stator resistance identification and speed estimation module.

\subsection{Rotor resistance and speed identification based on BEMF-MRAS}

Known from $[4,5]$, when the motor is in steady state, rotor resistance and rotor speed cannot be identified simultaneously in the RF-MRAS based system. Thus, rotor resistance and rotor speed only can be decoupled by adding additional incentives. In this paper, superposed a low frequency AC signal $i_{s d}^{*}$ on the given exciting current $i_{s d}$, it ensures the decoupling of rotor speed $\omega_{r}$ and rotor resistance $R_{r}$. In order to identify rotor resistance, it selects the current model with rotor resistance information as an adjustable model, and chooses the voltage model without rotor resistance as a reference model. Its selfadaptive law is:

$$
\begin{gathered}
\hat{R}_{r}=\left(k_{p}+k_{i} / s\right)\left(\hat{e}_{r \alpha} e_{r \beta}-e_{r \alpha} \hat{e}_{r \beta}\right) I_{s d}^{*} \\
I_{s d}^{*}=i_{s d}+i_{s d}^{*}
\end{gathered}
$$

\subsection{Speed identification algorithm based on stator- rotor resistance online identification}

Fig. 2 shows the MRAS speed identification system block diagram [6]. It is divided into two sub-modules. One is MRAS speed and rotor resistance identification. It has four inputs, which are stator voltage $u_{s}$, stator current $i_{s}$, low frequency exciting current signal $i_{s d}^{*}$ and stator resistance $R_{s}$ identified by MRAS stator resistance identification. The other sub-module is MRAS stator resistance identification. It also has four inputs, which are stator voltage $u_{s}$, stator current $i_{s}$, rotor resistance $R_{r}$ and rotor speed $\omega_{r}$ identified by MRAS speed and rotor resistance identification. According to section 2.2, MRAS speed and rotor resistance identification can identify the speed $\omega_{r}$ and rotor resistance $R_{r}$ correctly, as long as superposing a low frequency signal $i_{s d}^{*}$ on the given exciting current $i_{s d}$. Meanwhile, MRAS stator resistance

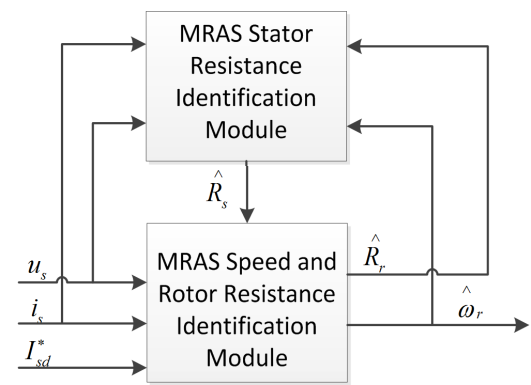

Fig. 2. Block diagram of speed identification system 


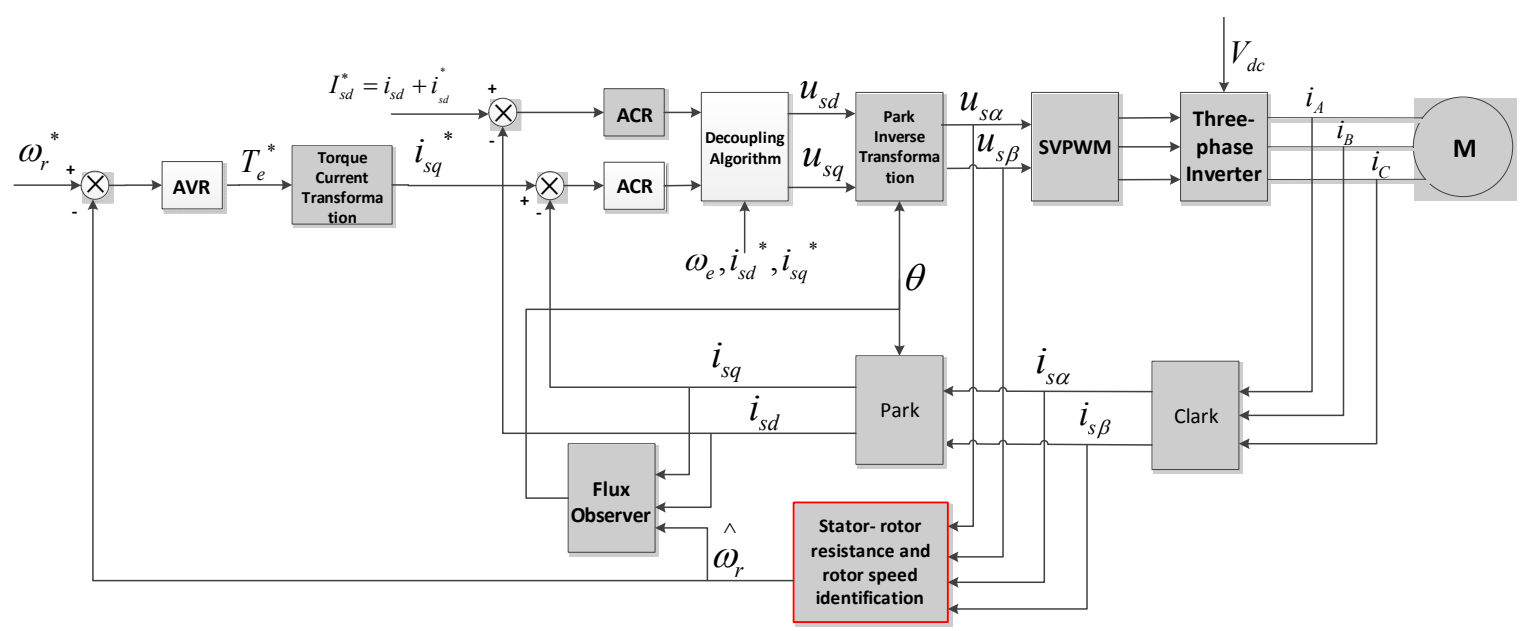

Fig. 3. Vector control system based on BMEF-MRAS with stator-rotor resistance and rotor speed identification

identification module uses the identified $\omega_{r}$ and $R_{r}$ as the inputs to estimate the stator resistance $R_{s}$. And $R_{s}$ also participates in the identification of $\omega_{r}$ and $R_{r}$. This mutual identification can improve the accuracy of estimated speed $\omega_{r}$.

\section{Vector Control System Based on MRAS with Stator-rotor Resistance and Speed Identification}

Fig. 3 shows the vector control system based on MRAS with stator-rotor resistance and rotor speed identification [7]. The difference between this vector control system and traditional one with sensor is that the rotor speed is estimated by MRAS replaces the one acquired from sensors. In addition, flux observer takes estimated speed as the input to observe flux; a low frequency AC signal $i_{s d}^{*}$ is superposed on the exciting current $i_{s d}$, and the MRAS module is the statorrotor resistance and rotor speed identification module mentioned above.

\section{Results of Simulink Simulation}

The induction motor parameters used in Simulink simulation model are shown in Table 1.

\subsection{The response speed of speed identification}

Next, the difference of response speed between sensorless vector control system based on RF-MRAS and that based on BEMF-MRAS will be analyzed. The analysis is conducted in two kinds of motor operating conditions, when running at $100 \mathrm{rpm}$ and $1000 \mathrm{rpm}$.

Fig. 4 (a) shows the simulation results of two kinds of the control algorithms, when $n=100 \mathrm{rpm}$. As it is shown, rotor speed of RF-MRAS reaches the given value at $t=2 \mathrm{~s}$, while rotor speed of BEMF-MRAS reaches the given value
Table 1. Parameters of the induction motor

\begin{tabular}{c|c|c|c|c}
\hline$U_{N}$ & $380 \mathrm{~V}$ & & $R_{r}$ & $5.22 \Omega$ \\
\hline$T_{e N}$ & $7.4 \mathrm{Nm}$ & & $L_{s}$ & $0.287 \mathrm{H}$ \\
\hline$P_{N}$ & $1.1 \mathrm{~kW}$ & & $L_{r}$ & $0.287 \mathrm{H}$ \\
\hline$f_{N}$ & $50 \mathrm{~Hz}$ & & $L_{m}$ & $0.25 \mathrm{H}$ \\
\hline$n_{N}$ & $1390 \mathrm{rpm}$ & & $J$ & $0.0021 \mathrm{~kg} \cdot \mathrm{m}^{2}$ \\
\hline$R_{s}$ & $4.0 \Omega$ & & $p$ & 2 \\
\hline
\end{tabular}
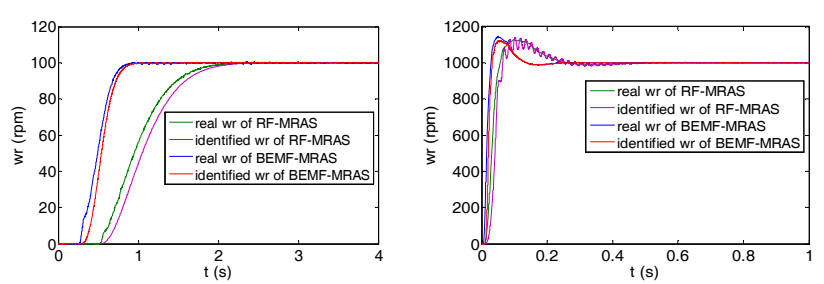

Fig. 4. Rotor speed simulation waveform of RF-MRAS and BEMF-MRAS

at about $t=1 \mathrm{~s}$. Fig. 4 (b) shows the simulation results of two control algorithms, when $n=1000 \mathrm{rpm}$. As it is shown, rotor speed of RF-MRAS reaches the given value at $t=0.3 \mathrm{~s}$, while rotor speed of BEMF-MRAS reaches the given value at $t=0.2 \mathrm{~s}$.

As the simulate result shown in Fig. 4, under the same condition, the response speed of speed identification based on BEMF-MRAS is faster than that based on RF-MRAS. The reason is that the speed identification based on BMEFMRAS does not contain the integral element, which accelerates the response speed. Meanwhile, it can reduce the time lag of the sensorless vector control system, and improve the stability and instantaneity of the control system.

4.2 The Steady State Accuracy of Speed Identification

When motor is running, the motor parameters such as stator resistance and rotor resistance will change slowly. In order to be more practical, the resistances in this paper are changing exponentially as follows: 


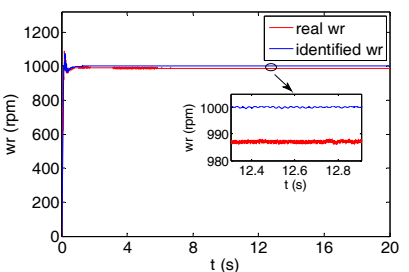

(a)

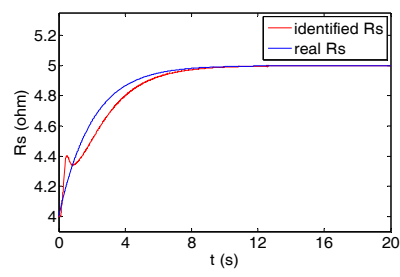

(c)

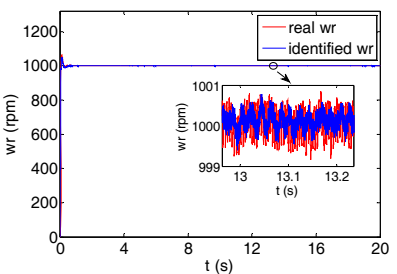

(b)

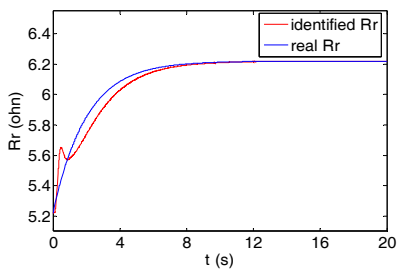

(d)
Fig. 5. Simulation waveform of the system when $n=1000$ rpm

$$
\begin{gathered}
\text { Stator resistance: } R_{S}=4+\left(1-e^{-\tau t}\right) \\
\text { Rotor resistance: } \quad R_{r}=5.22+\left(1-e^{-\tau t}\right)
\end{gathered}
$$

When $t=0, R_{s}=4 \Omega$ and $R_{r}=5.22 \Omega$ are rated values. When $t \rightarrow \infty, R_{s}=5 \Omega$ and $R_{r}=6.22 \Omega$. Meanwhile, the time constant $\tau$ is always set as 0.5 .

Fig. 5 shows the simulation waveform when motor is running at high speed of $1000 \mathrm{rpm}$, and the simulation takes 20 seconds. Fig. 5 (a) shows the rotor speed based on BEMF-MRAS without stator-rotor resistance identification modules, when stator resistance and rotor resistance are changing. Fig. 5 (b)-(d) show the simulation waveform based on BEMF-MRAS with stator-rotor resistance identification modules. According to Fig. 5 (a), when the motor is running at high speed, the stator resistance has a small voltage drop. Therefore, the resistance has little influence on the speed identification. And the error between real speed and given speed is small, which is about 1.5\%. As shown in Fig. 5 (b), with stator-rotor resistance identification modules, the rotor speed reaches the given speed at about $t=1 \mathrm{~s}$ and is stable at around 1000 $\mathrm{rpm}$. According to the partial enlarged detail view, the error of identified speed is less than $0.1 \%$. As shown in Fig. 5 (c) and (d), stator resistance reaches the stable value $5 \Omega$ at about $t=13 \mathrm{~s}$ and rotor resistance reaches the stable value $6.22 \Omega$ at about $t=12 \mathrm{~s}$. The stator-rotor resistance identification modules can follow the real value well, and the relative errors are respectively less than $1.5 \%$ and $2 \%$.

Fig. 6 shows the simulation waveform when motor is running at low speed of $100 \mathrm{rpm}$, and the simulation time is 20 seconds. Fig. 6 (a) shows the rotor speed based on BEMF-MRAS without stator-rotor resistance identification modules, when stator resistance and rotor resistance are changing. Fig. 6 (b) shows the rotor speed based on BEMF-MRAS with stator-rotor resistance identification

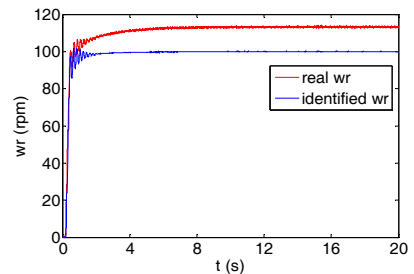

(a)

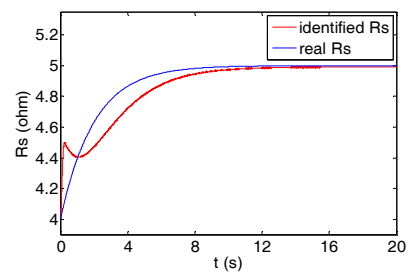

(c)

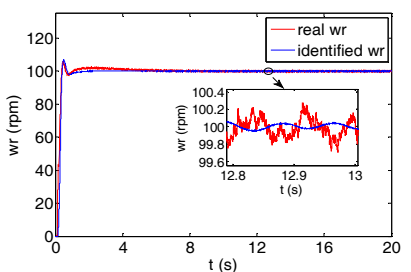

(b)

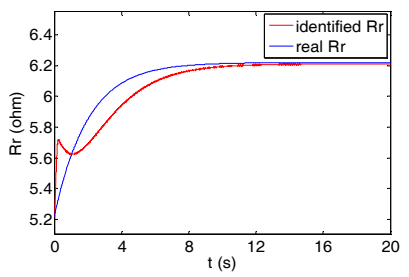

(d)
Fig. 6. Simulation waveform of the system when $n=100$ rpm

modules. According to Fig. 6 (a), when the motor is running at low speed, the voltage drop of stator resistance is equivalent to back electromotive force. So when the resistance changes, it cannot be neglected. Otherwise, the speed identification module cannot identify the rotor speed correctly. It will make the motor output speed fail to reach the given speed. And the error is large, which can be about $14 \%$ of the given speed. As shown in Fig. 6 (b), the rotor speed reaches the given speed at about $t=2 \mathrm{~s}$ and is stable at around $100 \mathrm{rpm}$. According to the partial enlarged detail view, the error of identified speed is less than $0.3 \%$. As shown in Fig. 6 (c) and (d), stator resistance reaches the stable value $5 \Omega$ at about $t=12 s$ and rotor resistance reaches the stable value $6.22 \Omega$ at about $t=12 s$. The statorrotor resistance identification modules can follow the real value well.

According to Fig. 5 and Fig. 6, when the motor is running at high speed and low speed, the error between real rotor speed and estimated speed is small in the steady state. The stator-rotor resistance identification modules can identify the stator and rotor resistance with high precision, which is less than $2 \%$. Thus, the accuracy of speed identification is ensured.

As shown in Table 2, the improved mutual speed identification based on BEMF-MRAS proposed by this paper is better than the traditional speed identification based on RF-MRAS in terms of response speed and steady

Table 2. Comparison of the simulation results of two control algorithms

\begin{tabular}{c|c|c|c}
\hline $\begin{array}{c}\text { Control } \\
\text { algorithm }\end{array}$ & Rotor speed & $\begin{array}{c}\text { regulation } \\
\text { time }\end{array}$ & $\begin{array}{c}\text { Steady-state } \\
\text { accuracy }\end{array}$ \\
\hline \multirow{2}{*}{ RF-MRAS } & $100 \mathrm{rpm}$ & $2.0 \mathrm{~s}$ & $0.5 \%$ \\
\cline { 2 - 4 } & $1000 \mathrm{rpm}$ & $0.3 \mathrm{~s}$ & $0.4 \%$ \\
\hline \multirow{2}{*}{ BEMF-MRAS } & $100 \mathrm{rpm}$ & $1.0 \mathrm{~s}$ & $0.3 \%$ \\
\cline { 2 - 4 } & $1000 \mathrm{rpm}$ & $0.2 \mathrm{~s}$ & $0.1 \%$ \\
\hline
\end{tabular}


state accuracy.

In summary, the simulation results above show that:

1) The proposed algorithm in this paper avoids the detrimental effect caused by integral element in RFMRAS, and speeds up the rotor speed identification.

2) Compared to the mutual speed identification based on BEMF-MRAS without stator-rotor resistance identifycation, the proposed algorithm in this paper significantly improves the accuracy of speed identification.

\section{Experimental Results}

Fig. 7 shows the experimental platform of induction motor vector control system. The controller is based on DSP2812 and the motor is $1.1 \mathrm{~kW}$ squirrel-cage induction motor. For the motor parameters, refer to Table 1. And all the PI parameters used are shown in Table 3.

Fig. 8 shows the rotor speed and current waveform, when the motor is running at low speed. According to Fig.

Table 3. PI parameters

\begin{tabular}{c|c|c}
\hline & $\mathrm{Kp}$ & $\mathrm{Ki}$ \\
\hline d-current loop & 25 & 10 \\
\hline q-current loop & 25 & 10 \\
\hline Speed loop & 0.08 & 0.5 \\
\hline Stator resistance identification & 0.001 & 3 \\
\hline Rotor resistance identification & 0.002 & 5 \\
\hline Speed identification & 0.001 & 10 \\
\hline
\end{tabular}

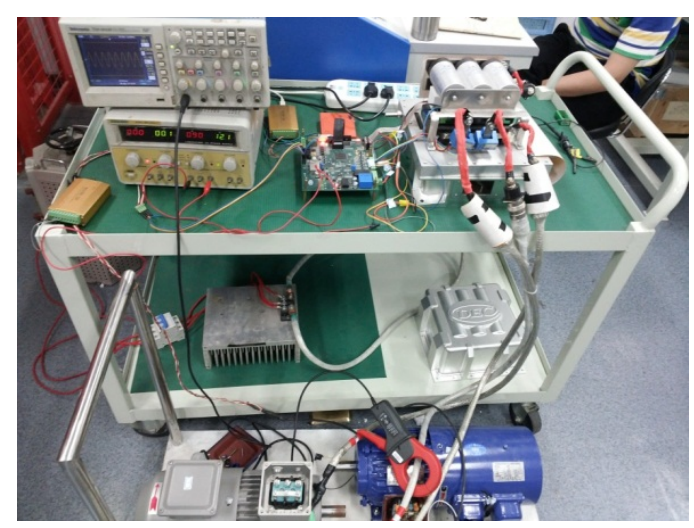

Fig.7. Experimental platform

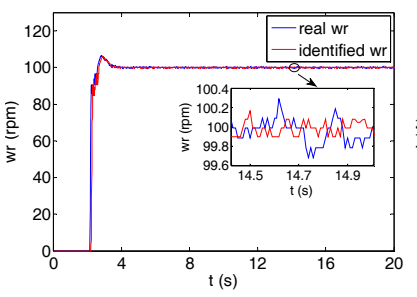

(a)

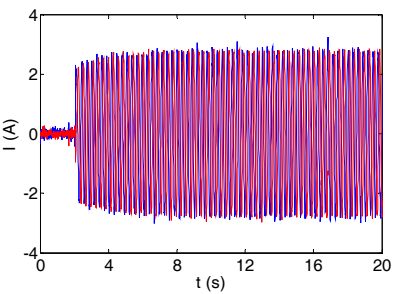

(b)
Fig. 8. Speed and current waveform when $n=100 \mathrm{rpm}$
Table 4. Comparison of the experimental results of two control algorithms

\begin{tabular}{c|c|c|c}
\hline Control algorithm & Rotor speed & Regulation time & $\begin{array}{c}\text { Steady-state } \\
\text { accuracy }\end{array}$ \\
\hline \multirow{2}{*}{ RF-MRAS } & $100 \mathrm{rpm}$ & $4.5 \mathrm{~s}$ & $<0.6 \%$ \\
\cline { 2 - 4 } & $1000 \mathrm{rpm}$ & $6 \mathrm{~s}$ & $<0.4 \%$ \\
\hline \multirow{2}{*}{ BEMF-MRAS } & $100 \mathrm{rpm}$ & $3.2 \mathrm{~s}$ & $<0.4 \%$ \\
\cline { 2 - 4 } & $1000 \mathrm{rpm}$ & $4 \mathrm{~s}$ & $<0.2 \%$ \\
\hline
\end{tabular}

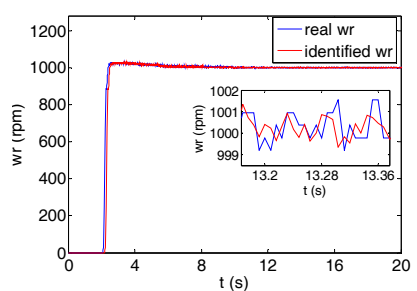

(a)

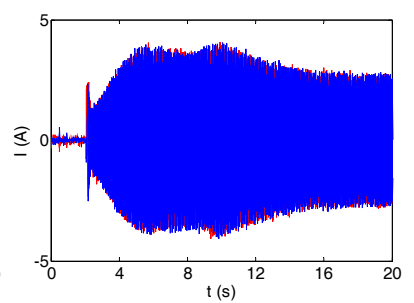

(b)
Fig. 9. Speed and current waveform when $n=1000 \mathrm{rpm}$

8 (a), when the given speed is $100 \mathrm{rpm}$, the motor starts at $t=2 s$, and after about $3 \mathrm{~s}$, the real speed and identified speed reach the given speed. They follow the given speed well, and the error of the identified speed and real speed are less than $0.4 \mathrm{rpm}$ when it is in steady state. According to (b), there is no distortion in stator current, and the waveform is sine wave.

Fig. 9 shows the rotor speed and current waveform, when the motor is running at high speed. According to (a), the motor starts at $t=2 s$, and after about $4 \mathrm{~s}$, the real speed and identified speed reach the given speed of 1000 rpm. In the steady state, the error of the identified speed and real speed are less than $2 \mathrm{rpm}$. According to (b), during the whole runtime, there is no obvious distortion in stator current, and the waveform is sine wave.

As shown in Fig. 8 and Fig. 9, no matter the motor is running at high speed or at low speed, the algorithm can identify the rotor speed with high precision, and the identified stator and rotor resistance can follow the real value well. Moreover, with this sensorless vector control algorithm, the stator current has no obvious distortion, and it can meet the requirement of high-performance control.

In Table 4, the regulation time and steady state accuracy of the speed identification based on RF-MRAS can be obtained by papers [8] and [9]. According to the Table 4, in terms of response speed and steady state accuracy, the algorithm proposed in this paper is obviously better than the traditional algorithm based on RF-MRAS. Thus, it verifies the feasibility and practicality of this algorithm in the motor experimental platform.

\section{Conclusion}

In this paper, an improved BEMF-MRAS based mutual speed identification algorithm is proposed. Compared with 
BF-MRAS based mutual speed identification, it speeds up the dynamic response. Compared with BEMF-MRAS based mutual speed identification, it improves the accuracy of speed estimation. In addition, its feasibility and effectiveness are verified through Simulink simulation and experiment in the motor experimental platform. It can track real-time change of stator-rotor resistance, estimate rotor speed with high precision rapidly, and meet static and dynamic requirements of sensorless vector control system well.

\section{References}

[1] Hui Ding, Xiehe Hu. The view of control strategy for induction motor drive system [J]. Journal of Zhejiang University(Engineering Science), 2011,01: pp. 50-58.

[2] Yiheng Chen, Ailing Zhang, Yihai Zhang. A method of speed identification based on improved BEMFMRAS [J]. Industry and Mine Automation, 2013, 09: pp. 75-79.

[3] Jian Gao, Shoudao Huang, Xiaofeng Ma, Guoyang Cai, Yanfei Cao. Sensorless vector control system for bearingless induction motor based on mutual MRAS[J]. Transactions of China Electrotechnical Society, 2008, 11: pp. 41-46.

[4] Mingyu Wang, Yangyu Chen, Wei Deng, Ruimiao Wang. The method of speed identification for induction motor with stator and rotor resistance online identification[J]. Electric Machines and Control, 2010, 04: pp. 66-71.

[5] Beguenane R, El Hachemi Benbouzid M, Tadjine M, et al. Speed and rotor time constant estimation via MRAS strategy for induction motor drives[C]. IEEE International Electric Machines and Drives Conference Record, 1997: B3/5.1-TB3/5.3.

[6] Wang Z, Zhang C. Speed identification about enhanced magnetism motor with MRAS[C]. Intelligent Control and Automation, 2008. WCICA 2008. 2008: pp. 7349-7354.

[7] Cirrincione M, Accetta A, Pucci M, et al. MRAS speed observer for high-performance linear induction motor drives based on linear neural networks[J]. IEEE Transactions on Power Electronics, 2013, 28(1): pp. 123-134.

[8] R. Ajabi Farshbaf, M.R. Azizian, A. Ebrahimi. NNMRAS based speed estimator VS. RF-MRAS one: design and comparison, Power Electronics and Drive Systems Technology (PEDSTC), 2012 3rd, pp. 223228.

[9] Ms. M. Nandhini Gayathri, S.Himavathi, R.Sankaran. Comparison of Rotor Flux and Reactive Power based MRAS Rotor Resistance Estimators for Vector Controlled Induction Motor Drive, International Journal of Engineering, vol. 25, No. 3, pp. 205-212.

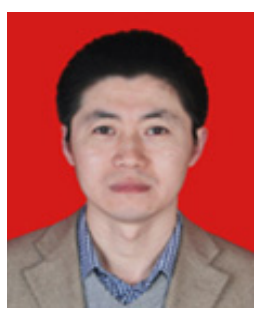

Hong Zheng $\mathrm{He}$ received $\mathrm{PhD}$. in control science and engineering from University of Electronic Science and Technology of China. His research interests are renewable energy system control and power electronics and motion control.

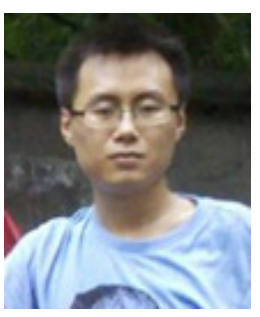

Jiancheng Zhao He received Bachelor's degree in electrical automation specialty from Central South University. $\mathrm{He}$ study for master's degree on control engineering in University of Electronic Sci\&Tech of China.

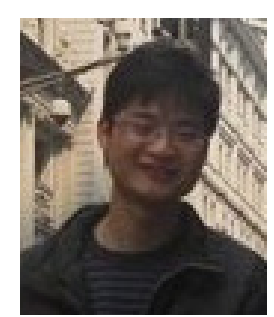

Liangzhong Liu He have got Master degree on control science and engineering in University of Electronic Sci $\&$ Tech of China. He works at Tenda corporation in Shenzhen. 